\title{
ANÁLISIS SOBRE LAS IMPLICACIONES DE LA NUEVA ESTRUCTURA DE LAS TITULACIONES OFICIALES: EL ESQUEMA “3+2"
}

\author{
Prof. Dr. Ángel Cobo Ortega \\ Defensor de la Universidad de Cantabria
}

I. Antecedentes: la adaptación del sistema universitario español al Espacio Europeo de Educación Superior

En octubre de 2007 se produce la aprobación del Real Decreto 1393/2007 por el que se establecía la ordenación de las enseñanzas universitarias oficiales, dando de esa manera el pistoletazo de salida para la adaptación efectiva del Sistema Universitario Español al Espacio Europeo de Educación Superior (EEES). En ese Real Decreto inicial se fijaba el curso 2010-2011 como curso en el que ya no podrían ofertarse plazas de nuevo ingresos para las titulaciones actuales en aquel momento. La fijación de este marco normativo daba por tanto a las Universidades 3 cursos académicos para rediseñar todas sus titulaciones, pasar por los correspondientes procesos de verificación, adaptar sus normativas internas, diseñar nuevos modelos pedagógicos,... en definitiva, un cambio profundo de nuestro sistema universitario con unos plazos de ejecución cortos con objeto de llegar al horizonte del 2010 marcado en la Declaración de Bolonia como fecha prevista para la plena consecución de los objetivos del EEES.

El Decreto 1393/2007 optó por fijar en 240 créditos la carga de las titulaciones de Grado (salvo los casos de titulaciones que habilitaban para determinadas profesiones) y definir una horquilla de entre 60 y 120 créditos para la configuración de los programas de Máster. En aquel momento, ya se oían voces contrarias a esta estructuración de las enseñanzas universitarias y, algunos de los argumentos, que se han utilizado recientemente, para justificar el cambio aprobado en febrero de 2015, ya se manejaban entonces para indicar que parecía más razonable ir a un modelo más flexible con grados de menor duración. No obstante, los responsables educativos en aquel momento optaron por un sistema en el que los grados tuvieran 4 años de duración.

Algunos argumentos que se oían para defender este modelo eran: 
1. Necesidad de favorecer una movilidad o reorientación de los estudios tras el primer año, configurando el primer año como de formación básica, equiparable en cierto modo a un "tercer curso de bachillerato" pero con una orientación hacia una gran rama de conocimiento. Con ese motivo se obligó a que los grados incluyeran 60 créditos de formación básica con materias definidas en cada rama.

2. $\quad$ En muchos de los países del EEES el acceso a los estudios universitarios se produce con más años de bachillerato o educación secundaria superior. Por ejemplo, en países nórdicos con muy buena valoración de sus sistemas educativos (Finlandia, Dinamarca, Noruega, Suecia) o países como Alemania los estudiantes acceden a la universidad con 19 años y por tanto una formación previa más sólida (ver Tabla 1).

\begin{tabular}{|c|c|c|c|}
\hline Reino Unido (Escocia) & 2 años (16-18) & Rumanía & 3 años (16-19) \\
\hline Holanda & 2 años (16-18) & Letonia & 3 años (16-19) \\
\hline Lituania & 2 años (16-18) & Estonia & 3 años (16-19) \\
\hline Malta & 2 años (16-18) & Chipre & 3 años (16-19) \\
\hline España & 2 años (16-18) & Austria & 4 años (14-18) \\
\hline Francia & 3 años (15-18) & Bélgica & 4 años (14-18) \\
\hline Grecia & 3 años (15-18) & República Checa & 4 años (14-18) \\
\hline Irlanda & 3 años (15-18) & Hungría & 4 años (14-18) \\
\hline Portugal & 3 años (15-18) & Reino Unido & 4 años (14-18) \\
\hline $\begin{array}{l}\text { Alemania (en la mayoría } \\
\text { de los Länder) }\end{array}$ & 3 años (16-19) & Luxemburgo & 4 años $(15-19)$ \\
\hline Finlandia & 3 años (16-19) & Liechenstein & 4 años (15-19) \\
\hline Dinamarca & 3 años (16-19) & República Eslovaca & 4 años $(15-19)$ \\
\hline Suecia & 3 años (16-19) & Islandia & 4 años $(16-20)$ \\
\hline Noruega & 3 años (16-19) & Bulgaria & 5 años (14-19) \\
\hline Polonia & 3 años (16-19) & Italia & 5 años (14-19) \\
\hline
\end{tabular}

Tabla 1. Duración de los estudios equivalentes al bachillerato en diferentes países europeos (Fuente Comisión Europea, Eurydice y euroeducation.net)

3. El diseño propuesto trataba también de favorecer la incorporación a los estudios universitarios de estudiantes procedentes de la educación superior no universitaria 
(ciclos formativos de grado superior). De hecho se instaba a las universidades a un reconocimiento importante de créditos a los estudiantes procedentes de estos estudios.

4. $\quad$ No seguir reduciendo la carga de las titulaciones. Muchas titulaciones habían ajustado ya sus planes de estudio a 4 años desde anteriores licenciaturas o ingenierías de 5 años. Sí que es cierto que en muchos casos el ajuste había resultado complejo y se había limitado a "comprimir" asignaturas y créditos.

5. $\quad$ En algunos casos además se había aprovechado para poner en marcha planes piloto de adaptación metodológica al EEES plenamente integrados en titulaciones de cuatro años de duración.

6. Apostar en aquel momento por un sistema "3+2" parecía a algunos dar un paso atrás, volviendo al sistema de licenciaturas divididas en dos ciclos.

7. Finalmente, se insistía mucho en que la adaptación al EEES no suponía homogeneizar los sistemas educativos, se hablaba más bien de armonizar o sintonizar (tunning) y, por tanto, se argumentaba que no era necesario ir a un modelo común en todos los países del EEES.

Desde su aprobación, el RD 1393/2007 ha sufrido ya varias modificaciones, de mayor o menor calado, pero en general sin afectar a la estructura general de las titulaciones, hasta llegar al último cambio realizado. La primera modificación se produce con el Real Decreto 861/2010 en el que se realizan algunas modificaciones en relación a la posibilidad de configurar títulos interuniversitarios; se posibilita el reconocimiento de créditos de enseñanzas superiores no universitarias, experiencia laboral o títulos propios, pero imponiendo algunas restricciones; se permite que los Grados definan menciones o intensificaciones y los programas de Máster especializaciones; se determina que el reconocimiento de créditos por actividades culturales sea de al menos 6 créditos en lugar de un máximo de 6 ; se establece la posibilidad de regulación específica de Grados no habilitantes pero que son requisito para acceso a Másteres habilitantes; se obliga al reconocimiento de la menos 36 créditos básicos de la rama cuando se producen cambios de titulaciones dentro de una misma rama; se actualiza la información relacionada con los accesos a la universidad; necesidad de adscripción de los títulos de Máster a una rama de conocimiento; se establece alguna limitación y regulación sobre los créditos de complementos formativos para acceso al Máster; se producen algunos cambios en los procesos de acreditación y verificación, así como en el registro de títulos en el RUCT; se obliga a garantizar al menos cuatro convocatorias de exámenes para los estudiantes en planes en extinción; finalmente se modifica el modelo de memoria de verificación. 
Como consecuencia de la regulación específica de las enseñanzas oficiales de Doctorado, en el Real Decreto 99/2011 se realizaron ajustes en el Artículo 11 del RD 1393/2007 y se suprimió el Capítulo V del Decreto, el relacionado con las enseñanzas de Doctorado, y un apartado del modelo de la memoria de verificación.

Durante los años de vigencia de este Decreto, se han tenido que realizar además dos modificaciones para ampliar los plazos para la renovación de la acreditación de los títulos oficiales, modificaciones que corresponden a los Decretos 534/2013 y 967/2014.

En el Real Decreto 96/2014 se produce otra modificación importante para reconocer el nivel de Máster (nivel 3 del MECES) para aquellos títulos de Grado de al menos 300 créditos ECTS que comprendan al menos 60 créditos de Nivel de Máster, y que obtengan este nivel de cualificación mediante resolución del Consejo de Universidades.

Y de esta manera se llega a la última modificación, la aprobada a través del Real Decreto 43/2015, de 2 de febrero, modificación que ha sido objeto de críticas desde diferentes sectores y que será objeto de análisis detallado más adelante.

\section{Situación actual del sistema universitario español}

Después de 7 años de la aprobación del Real Decreto 1393/2007 y tras el esfuerzo de adaptación de todas las titulaciones ofertadas por las universidades españolas, la situación actual es la que trata de presentarse a continuación.

La desaparición del catálogo de titulaciones y su sustitución por el registro de títulos ha provocado que, globalmente el Sistema Universitario Español tenga una amplísima oferta de titulaciones diferentes, en algunos casos incluso puede parecer excesiva. Es difícil dar un número exacto de titulaciones ofertadas actualmente ya que el dato varía según la fuente consultada, pero como referencia se pueden tomar los valores obtenidos en tres fuentes oficiales: el RUCT ${ }^{1}$ (Registro de Universidades Centros y Títulos), el servicio QEDU² (Qué Estudiar y Dónde en la Universidad) del Ministerio de Educación y el buscador de títulos de la ANECA $^{3}$. Los números de titulaciones registradas en esas fuentes son los que se muestran en la Tabla 2.

\footnotetext{
${ }^{1}$ https://www.educacion.gob.es/ruct/consultaestudios?actual=estudios

${ }^{2}$ https://www.educacion.gob.es/notasdecorte/busquedaSimple.action

${ }^{3}$ http://srv.aneca.es/ListadoTitulos/
} 


\begin{tabular}{c|cc}
\hline Fuente & Número de títulos de Grado & Número de títulos de Máster \\
\hline RUCT Servicio & 2672 & 5837 \\
QEDU & 3116 & 4757 \\
$\begin{array}{c}\text { Buscador de títulos de la } \\
\text { ANECA }\end{array}$ & 2743 & 4401 \\
\hline
\end{tabular}

Tabla 2. Número de titulaciones oficiales registradas en fuentes oficiales (fecha de consulta: 13 de abril de 2015)

Todas las titulaciones de grado están configuradas con 240 créditos ECTS, salvo aquellas que habilitan para profesiones que tienen regulaciones específicas que, exigen una carga docente de más créditos. En el caso de los programas de Máster, aunque el Real Decreto 1393 permite configurarlos con uno o dos años (hasta 120 créditos), la realidad es que el modelo más frecuente en nuestras universidades es el de Máster de 60 créditos. Incluso en programas de Máster que habilitan para profesiones reguladas de Ingeniero Superior nos podemos encontrar con Másteres de 60 créditos como continuidad de grados de ingeniería sin atribuciones y orientados específicamente hacia el acceso al Máster ("grados blancos”). Analizando los créditos de los diferentes programas que se incluyen en el servicio QEDU, la distribución del número de títulos en función de sus créditos es la que muestra la Tabla 3.

\begin{tabular}{|c|c|c|c|c|c|}
\hline Programas de Grado & № tit. & $\%$ & Programas de Máster & № tit. & $\%$ \\
\hline De 240 créditos & 2.971 & $95,4 \%$ & De 60 créditos & 3.542 & $74,6 \%$ \\
\hline Entre 300 y 359 créditos & 98 & $3,1 \%$ & Entre 61 y 119 créditos & 779 & $16,4 \%$ \\
\hline De 360 créditos & 44 & $1,4 \%$ & De 120 créditos & 425 & $9,0 \%$ \\
\hline
\end{tabular}

Tabla 3: Número de créditos por titulación en el servicio QEDU

Respecto al número de estudiantes matriculados en el Sistemas Universitarios Español, si se toma como referencia el último informe publicado por el Ministerio sobre "Datos Básicos del Sistema Universitario Español”4, correspondiente al curso 2013-2014, el número de estudiantes matriculados en estudios de Grado, más los que aún permanecen en titulaciones antiguas de primer y segundo ciclo, se estimaba en 1.438.115; mientras que en el caso de los programas de Máster ese número era de 109.113, observándose una cierta

\footnotetext{
${ }^{4}$ http://www.mecd.gob.es/dms/mecd/educacion-mecd/areas-educacion/universidades/estadisticas-informes/ datos-cifras/DATOS_CIFRAS_13_14.pdf
} 
estabilización con respecto a años precedentes. La evolución de esos números puede verse en

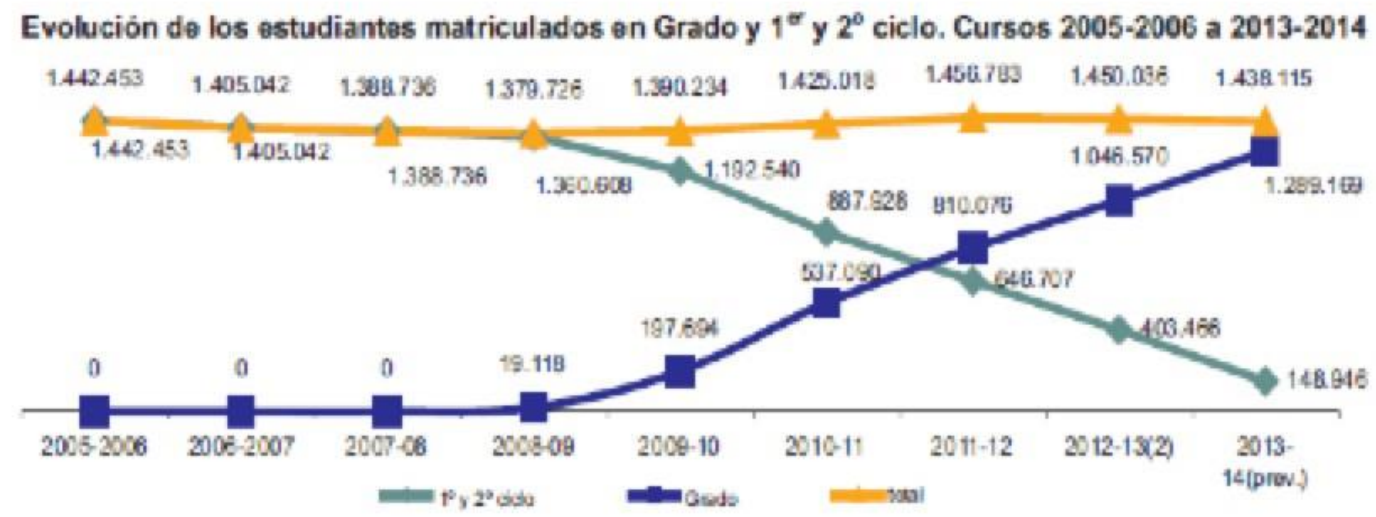

las Figuras 1 y 2.

Figura 1: Estudiantes matriculados en grado, primer y segundo ciclo (Fuente: Ministerio de

Educación, Datos Básicos del Sistema Universitario Español, curso 2013-2014)

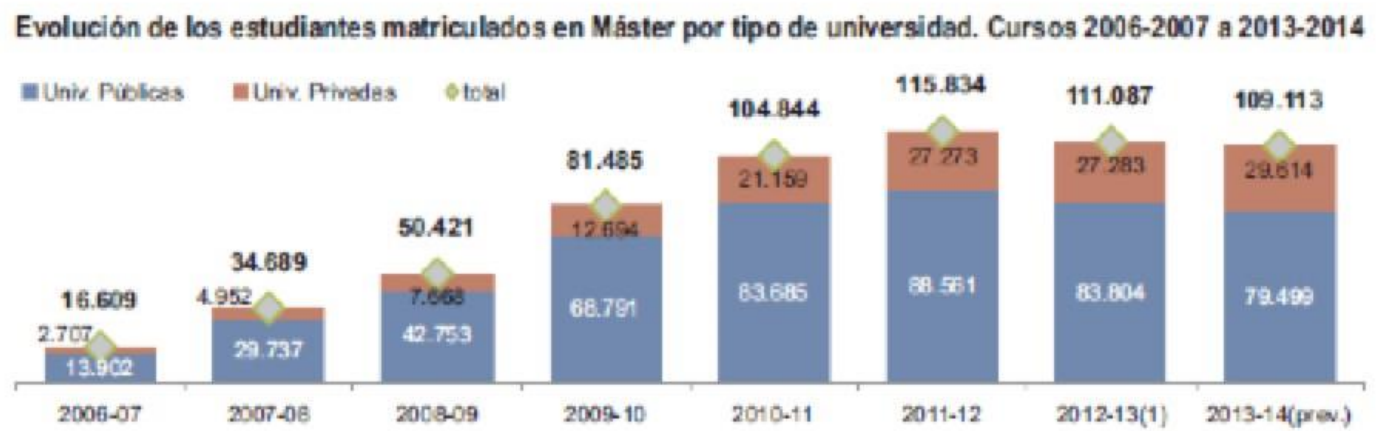

Figura 2: Estudiantes matriculados en Máster (Fuente: Ministerio de Educación, Datos Básicos del Sistema Universitario Español, curso 20132014)

Teniendo en cuenta esos números, y la oferta global de titulaciones, se podría hacer un sencillo cálculo del número promedio de estudiantes por titulación, pero tomando el resultado con la cautela que exige una estimación tan simplificada. Debe tenerse en cuenta que en los números de plazas de ingreso a las diferentes titulaciones hay una gran dispersión entre las 
diferentes áreas. De esta manera, si dividimos los 1.438.115 estudiantes de Grado o similar entre las 3.116 titulaciones de grado ofertadas en QEDU el promedio sería de 461,5 estudiantes por grado. En el caso de Máster, ese mismo cálculo conduciría a un promedio de 22,9 estudiantes por título de Máster. En el caso de los Másteres debe tenerse en cuenta, además, que un porcentaje destacado de esos estudiantes está formado por los que cursan programas que habilitan para el ejercicio de determinadas profesiones reguladas, por ejemplo Ingenierías Superiores.

En cuanto al reparto de los estudiantes por ramas de conocimiento y por tipo de universidad, la situación es la que muestra la Tabla 4. Como puede apreciarse, un 88,45\% de los estudiantes de Grado lo hacen en universidades públicas. En el caso de los estudios de Máster la diferencia entre universidades públicas y privadas es menor. También se observan diferencias significativas en función de las grandes áreas de conocimiento.

\begin{tabular}{lcccccccc}
\hline & \multicolumn{2}{c}{ Univ. públicas } & \multicolumn{2}{c}{ Univ. privadas } & Univ. públicas & \multicolumn{2}{c}{$\begin{array}{c}\text { Univ. } \\
\text { privadas }\end{array}$} \\
\hline Rama & Grado & $\%$ & Grado & $\%$ & Máster & $\%$ & Máster & $\%$ \\
\hline $\begin{array}{l}\text { C. Sociales y } \\
\text { Jurídicas }\end{array}$ & 580.648 & $85,83 \%$ & 95.847 & $14,17 \%$ & 38.406 & $63,6 \%$ & 21.994 & $36,4 \%$ \\
\hline $\begin{array}{l}\text { Ingeniería y } \\
\text { Arquitectura }\end{array}$ & 278.735 & $93,44 \%$ & 19.572 & $6,56 \%$ & 13.996 & $82,5 \%$ & 2.964 & $17,5 \%$ \\
\hline $\begin{array}{l}\text { Artes y } \\
\text { Humanidades }\end{array}$ & 137.138 & $95,93 \%$ & 5.816 & $4,07 \%$ & 11.241 & $89,9 \%$ & 1.259 & $10,1 \%$ \\
\hline $\begin{array}{l}\text { Ciencias de la } \\
\text { Salud }\end{array}$ & 193.768 & $81,80 \%$ & 43.121 & $18,20 \%$ & 9.739 & $77,6 \%$ & 2.805 & $22,4 \%$ \\
\hline Ciencias & 81.758 & $97,95 \%$ & 1.712 & $2,05 \%$ & 6.117 & $91,2 \%$ & 592 & $8,8 \%$ \\
\hline Total & 1.272 .047 & $88,45 \%$ & 166.068 & $11,55 \%$ & 79.499 & $72,9 \%$ & 29.614 & $27,1 \%$ \\
\hline
\end{tabular}

Tabla 4: Distribución del número de estudiantes de Grado y Máster entre universidades públicas y privadas.

El aspecto económico está claramente presente a la hora de analizar las consecuencias de la aplicación del Decreto recién aprobado. La situación actual de los precios públicos que se aplican a las titulaciones oficiales en las Universidades públicas se caracteriza por la existencia de diferencias notables de unas Comunidades Autónomas a otras y los importantes aumentos en los precios de segundas y sucesivas matrículas, tanto en los estudios de Grado como de Máster.

Otra característica común es la diferenciación de precio para los másteres en función de si habilitan o no para el ejercicio de determinadas profesiones reguladas, penalizando a los estudiantes de másteres no habilitantes. En concreto se observa un incremento medio del 46\% del precio del Máster habilitante con respecto al Grado, mientras que en los másteres no 
habilitantes ese incremento supone un 118\%. En concreto, los precios medios por crédito en primera matrícula durante el curso 2013-2014 fueron los que recoge la Tabla 5.

\begin{tabular}{lrrr}
\hline & Grado & $\begin{array}{c}\text { Master } \\
\text { habilitante }\end{array}$ & $\begin{array}{c}\text { Máster no } \\
\text { habilitante }\end{array}$ \\
\hline Precio medio & $\mathbf{1 8 , 4 2}$ & $\mathbf{2 6 , 8 9}$ & $\mathbf{4 0 , 1 1}$ \\
\hline Andalucía & 12,62 & 19,5 & 41,5 \\
\hline Aragón & 19,75 & 19,8 & 45,88 \\
\hline Asturias (Ppdo. de) & 17,13 & 26,54 & 33,45 \\
\hline Balears (llles) & 17,92 & 28,28 & 30,61 \\
\hline Canarias & 15,21 & 26,92 & 35,15 \\
\hline Cantabria & 13,5 & 22,85 & 36,56 \\
\hline Castilla y León & 23,11 & 32,45 & 41,17 \\
\hline Castilla-La Mancha & 15,24 & 27,64 & 29,25 \\
\hline Cataluña & 33,52 & 40,88 & 65,41 \\
\hline Gomunitat Valenciana & 20,39 & 20,39 & 46,2 \\
\hline Extremadura & 14,74 & 21,67 & 32,75 \\
\hline Galicia & 11,89 & 25,03 & 26,49 \\
\hline Madrid (Com. de) & 30,33 & 42,75 & 65 \\
\hline Murcia (Región de) & 15,58 & 25,53 & 44,22 \\
\hline Navarra (C. Foral de) & 19,22 & 27,7 & 44,32 \\
\hline País Vasco & 16,81 & 17,66 & 31,61 \\
\hline Rioja (La) & 18,37 & 19,62 & 39,52 \\
\hline U.N.E.D. & 16,18 & 38,89 & 32,93 \\
\hline & & &
\end{tabular}

Tabla 5: Precios públicos de Titulaciones en Universidades Públicas (primera matrícula) en el Curso 2013-2014.

Uno de los principales argumentos, utilizados por el Ministerio para justificar la necesidad de la reforma en la estructura de las titulaciones es, la equiparación a la mayor parte de los países del EEES y facilitar el reconocimiento efectivo de las titulaciones impartidas por las universidades españolas. Con objeto de analizar la situación global en Europa, se podría tomar como referencia el informe "El Espacio Europeo de Educación Superior en 2012: Informe sobre la implantación del Proceso de Bolonia” publicado por la Agencia Ejecutiva en el Ámbito Educativo, Audiovisual y Cultural (EACEA P9 Eurydice) ${ }^{5}$. En dicho informe se llega a las siguientes conclusiones:

Analizando la estructura de los Grados en los diferentes países del EEES se comprueba que efectivamente no existe un modelo único de programas de primer ciclo en el EEES. La mayoría de los países ofrecen una combinación de programas de 180 créditos ECTS, de 240 ECTS y de otra duración. El modelo de programas de grado de 180 créditos ECTS puede encontrarse únicamente en la Comunidad flamenca de Bélgica, en Francia, Italia, Liechtenstein y Suiza. También prevalece el modelo de 180 créditos ECTS -con más del

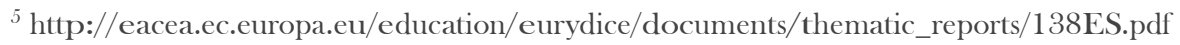


75\% de los programas- en otros 15 sistemas del EEES. En algunos países -Armenia, Chipre, Georgia, Kazajstán, Turquía y Ucrania- existe un modelo único de 240 créditos ECTS, que también predomina en más del 75\% de los programas de Azerbaiyán, Bosnia Herzegovina, Bulgaria, Grecia, España y Letonia. También debería incluirse a los Países Bajos en este grupo, dado que, aunque su porcentaje de programas de 240 créditos ECTS ronda el 45\%, el porcentaje de alumnos matriculados en ellos representa el 70\%. Todos estos datos se resumen en la Figura 3.

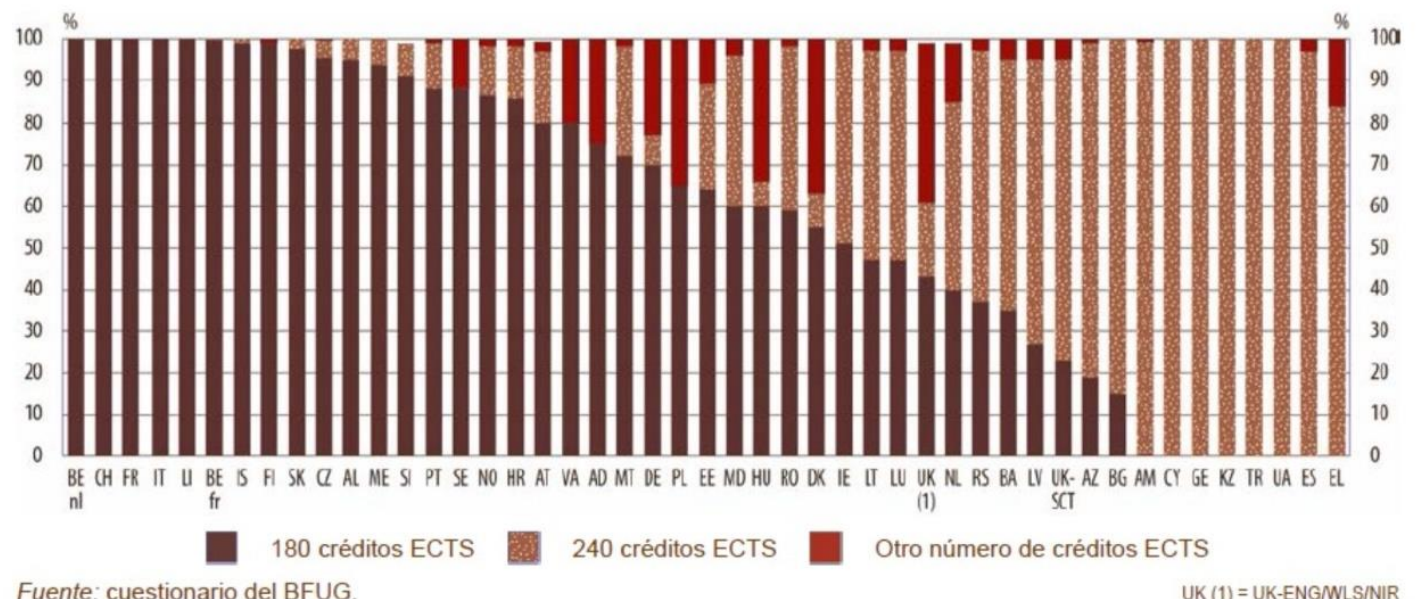

Figura 3: Estructura de las titulaciones de Grado en el EEES (Fuente: EACEA P9 Eurydice).

En el caso de los programas de Máster (ver Figura 4) el modelo más extendido es, con diferencia, el de 120 créditos ECTS, presente en 42 sistemas de educación superior. Este es el único modelo o el utilizado por más del 75\% de los programas en 26 páses. El modelo de 60 a 75 créditos ECTS se puede encontrar en 27 países y es el más frecuente en otros ocho, entre ellos España.

En definitiva, no existe un modelo único dentro del EEES ni para los programas de primer ciclo ni para los de segundo: en el primer ciclo, la mayoría de los países presentan una combinación de programas de 180 y 240 créditos ECTS y/o de otra duración. En segundo ciclo, el formato más común es del de los 120 créditos ECTS. El modelo $180+120$ créditos ECTS (“3+2”) es, por tanto, el más extendido, pero también se dan otro tipo de combinaciones dentro del EEES. 


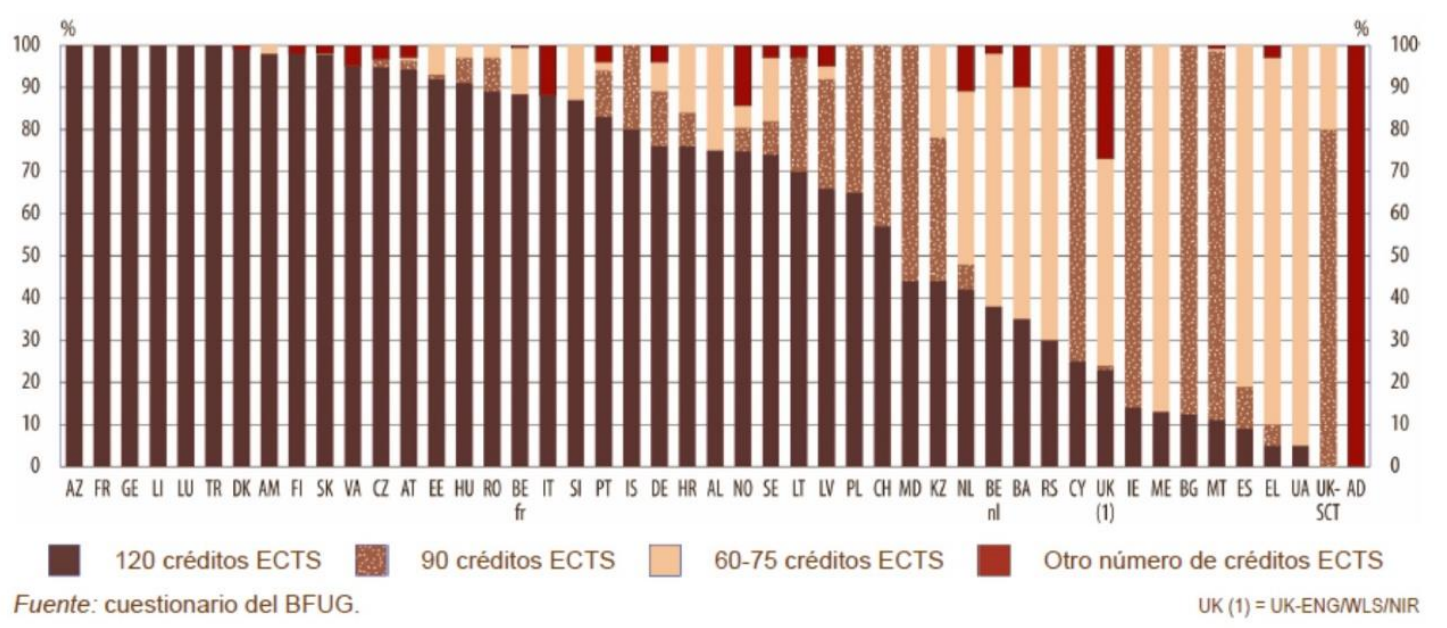

Figura 4: Estructura de las titulaciones de Máster en el EEES (Fuente: EACEA P9 Eurydice)

Además, de información sobre la estructura de las titulaciones, del informe de la EACEA se podrían extraer algunos otros datos que pueden ser interesantes para realizar un análisis. Por ejemplo el porcentaje de estudiantes que tras completar sus estudios de primer nivel (Grado) continúan con estudios de Máster. La Figura 5 muestra el porcentaje de estudiantes de primer ciclo que continúan sus estudios en un programa de segundo ciclo tras obtener el título (en un plazo de dos años), los datos se refieren al curso 2010/11. En el caso de España hay que tener en cuenta que en el curso 2010/2011 aún no se había producido ninguna graduación de titulados de acuerdo al nuevo sistema de Grados, salvo quizás los provenientes de cursos de adaptación al Grado, por tanto, los datos anteriores habría que tomarlos con cautela.

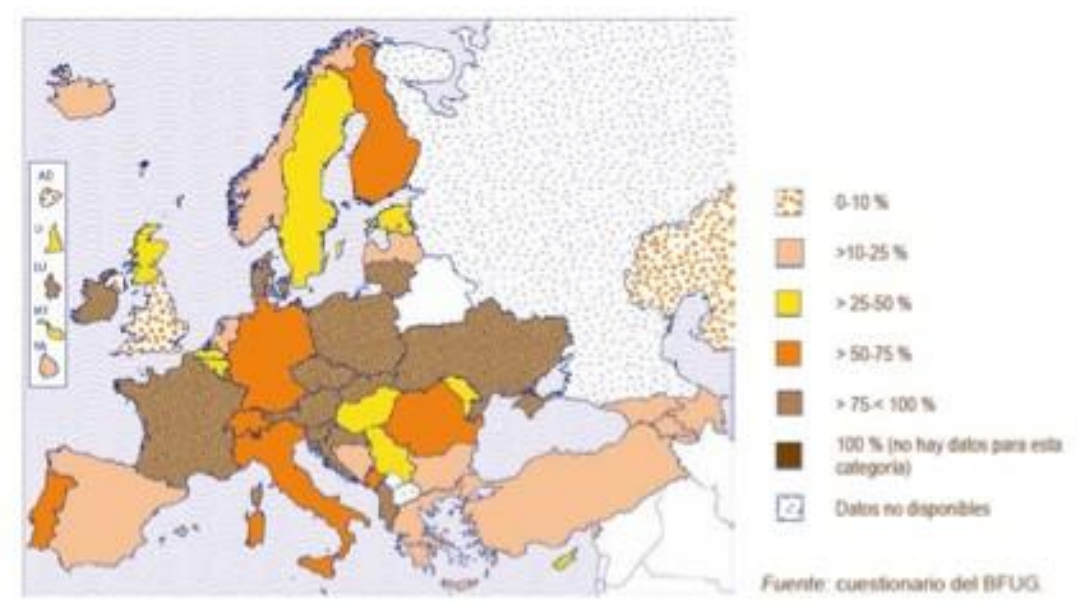


Figura 5: Porcentaje de estudiantes de primer ciclo (Grado) que continúan sus estudios en un programa de Máster (Fuente: EACEA P9 Eurydice).

De todos modos, tomando los datos de estudiantes matriculados del último informe del Ministerio correspondiente al curso 2013-2014, en el que se fijaba el número de egresados de titulaciones de Grado y similar en 208.317 estudiantes, y con la estimación de 109.113 estudiantes matriculados en Máster en el curso 2013-2014, parece que no llegamos al nivel de continuación de estudios en el nivel superior que se produce en otros países de nuestro entorno. El propio Ministerio estima en un 20\% el número de estudiantes que después de obtener el Grado optan por completar su formación con un Máster. Debe tenerse en cuenta, además, que una buena parte de los alumnos de Máster cursan programas de Máster que habilitan para el acceso a determinadas profesiones, por ejemplo, Ingenierías Superiores.

\section{Análisis del Real Decreto 43/2015 por el que se modifican determinados aspectos del Real Decreto 1393/2007}

El Real Decreto publicado el pasado 2 de febrero viene a plantear importantes cambios en la estructuración de las titulaciones oficiales. En la introducción del texto se viene a justificar la necesidad de este cambio con objeto de "garantizar la internacionalización de los estudiantes y nuestros egresados universitarios”. También se plantea la necesidad de homogeneizar, en los casos que sean conveniente, la duración de los estudios con la de países de nuestro entorno. En esa introducción al Decreto se indica, además, algo que deja abierta la posibilidad a cambios en la estructuración de los grados que habiliten para profesiones reguladas, se dice que, en esos casos "el Gobierno establecerá las condiciones a las que deberán adecuarse los correspondientes planes de estudio que, deberán ajustarse, en su caso, a la normativa europea aplicable”.

Se analizan a continuación los principales cambios que plantea este Decreto y sus posibles implicaciones.

En primer lugar, parece claro que un primer objetivo es buscar Grados de carácter más generalista y que la especialización se produzca realmente en el Máster. Así la modificación que se realiza del Artículo 3 del RD 1393/2007 es simplemente para incluir una declaración expresa de que "las Agencias de Evaluación tendrán en cuenta, a la hora de verificar y acreditar los títulos, que las propuestas de las Universidades primen los contenidos generalistas y de formación básica en los planes de estudios de títulos de Grado y los contenidos especializados en los planes de estudios de títulos de Máster”. Esa misma idea de 
grados generalistas y de no especialización se repite a la hora de modificar el Artículo 12. Es precisamente la modificación de ese Artículo 12 la que más críticas ha planteado en la comunidad universitaria, ya que dicha modificación abre las puertas a la posibilidad de definir planes de estudio de Grado con una carga de entre 180 y 240 créditos. Al permitir grados de 180 créditos ECTS se deben ajustar de manera proporcional determinados parámetros (créditos de formación básica, porcentaje de créditos básicos vinculados a materias del anexo, créditos de prácticas externas, trabajo fin de grado,...). El modelo se plantea como flexible, pudiendo voluntariamente las universidades optar por definir grados de 180 créditos ECTS. En caso de que opten por grados de menos de 240 créditos deberán arbitrar "mecanismos que complementen el número de créditos de Grado con el número de créditos de Máster, de manera que se garantice que la formación del Grado es generalista y los contenidos del Máster se orienten hacia una mayor especialización”.

A la hora de regular el reconocimiento de créditos, un aspecto que cambia es el reconocimiento de créditos de la misma rama (Artículo 13, párrafo a). Antes de la modificación a un estudiante que se cambiaba de estudios dentro de la misma rama se le reconocían al menos 36 créditos correspondientes a materias de formación básica de la rama, ahora podrían llegar a ser sólo el $15 \%$ de 45 créditos (6,75 créditos). De esta manera, en algunos casos, se dificultaría la reorientación de los estudios ante una mala elección inicial por el estudiante.

En lo que se refiere a los programas de Máster, el Decreto no plantea ningún cambio en su estructura, seguirán pudiéndose definir con planes de estudio de entre 60 y 120 créditos. De todos modos, a la hora de modificar el Artículo 15 se vuelve a insistir en el objetivo fundamental de especialización de los programas de Máster.

Los cambios planteados en la estructura de titulaciones de Grado tienen también su implicación en el acceso al Doctorado, de manera que se produce también un cambio en el Real Decreto 99/2011 por el que se regulan las enseñanzas oficiales de doctorado. En concreto, en el caso del acceso al Doctorado los nuevos graduados de 180 créditos ECTS tendrán que necesariamente cursar un Máster de 120 créditos ya que se exigirá superar al menos 300 créditos entre Grado y Máster.

Finalmente, el RD 43/2015 incluye disposiciones adicionales, transitorias y finales. En especial conviene tener presente la disposición adicional por la que se establece que todas las medidas planteadas no podrán suponer incremento de dotaciones, retribuciones ni gastos de personal. La disposición transitoria marca el proceso de transformación o extinción de estudios en los casos en los que un grado pase a ser de 180 créditos ECTS. Se fija el criterio de un mínimo de 4 
convocatorias para que los estudiantes terminen sus estudios de acuerdo a la organización por la que comenzaron.

\section{Reacciones ante la aprobación de los cambios en la estructura de los grados}

Obviamente, como en cualquier cambio propuesto, se pueden oír voces a favor y en contra con argumentaciones diferentes. Por parte del Ministerio se defiende la oportunidad y conveniencia del cambio con los siguientes argumentos. En la gran mayoría de países del EEES hay grados de 3 años o se combinan grados 3 y 4 años. Se argumenta también la dificultad actual de algunos egresados españoles para el acceso a programas de Doctorado en Europa por haber cursado másteres de 60 créditos. Para algunos el actual modelo universitario español es una distorsión frente al panorama universitario internacional y con la reestructuración propuesta se facilita que los españoles cursen un doctorado en el extranjero sin tener que ampliar sus estudios de máster; además los extranjeros que vengan a España con un grado de 180 créditos hecho también podrán adaptarse mejor. El Ministerio también destaca que el nuevo sistema plantea un modelo flexible al que las universidades voluntariamente pueden acogerse. No garantiza más homogeneidad entre las universidades (una misma titulación podría tener duraciones diferentes en dos universidades), pero lo mismo ocurría antes de la implantación del Plan Bolonia, con licenciaturas que en algunas universidades eran de 5 años y en otras de 4 . La capacidad de atracción de estudiantes por las universidades debería basarse en parámetros de calidad y no necesariamente en la duración de los estudios. Además de todo ello el Ministerio recuerda que las propias universidades solicitaban en su momento más flexibilidad a la hora de configurar las titulaciones, remitiéndose a las conclusiones de la Comisión Académica Sectorial de las Universidades Españolas (CASUE), de 17 y 18 de octubre de 2006 en las que se pedía optar por "una posición más flexible en la que existieran Grados entre 180 y 240 créditos".

Resulta paradójico que el argumento de coste económico para las familias sea utilizado tanto para defender la propuesta como para manifestarse abiertamente en contra. Por parte del Ministerio se hace una estimación de ahorro de 150 millones de euros para las familias por el paso de grados de 4 a 3 años. Para esa estimación el Ministerio se basa en que actualmente únicamente un 20\% de los graduados opta por seguir formándose en Máster, el Ministerio considera que esta situación no necesariamente debería cambiar con el nuevo esquema. Además se argumenta que, generalmente el acceso al Máster se produce tras haberse incorporado al mercado laboral. El Ministerio sostiene que "solo en el 10\% de los 
supuestos" se pide un Máster para acceder a un trabajo. En los propios planes del Ministerio se asume una reducción de estudiantes en las Universidades al considerar que el 80\% de ellos no continuarán un Máster y, en consecuencia, podrían pasar un año menos en la universidad. Realmente esos números de continuación de estudios en el nivel superior no son los que se producen en países de nuestro entorno, y pueden resultar un tanto contradictorios con la idea varias veces repetida en el Decreto de modificación, del carácter generalista de los Grados, dejando la especialización para los Másteres.

Antes de analizar las reacciones y opiniones de la comunidad universitaria ante los cambios aprobados, puede ser interesante recoger algunas de las afirmaciones y conclusiones del Consejo de Estado en su Dictamen $1146 / 2014^{6}$ a la vista del borrador previo a la aprobación del Real Decreto 43/2015. En primer lugar el Consejo de Estado establece que, una falta de estabilidad en la regulación de las enseñanzas durante los últimos años no parece beneficiar a la consecución de una educación de calidad en España y, declara que sería deseable alcanzar un acuerdo general de las fuerzas políticas y sociales para dar más estabilidad al sistema. Llama la atención también que, la flexibilidad que introduce la norma, puede generar disfunciones por la existencia de un mismo título con duraciones diferentes en dos universidades. El Consejo de Estado considera razonable que haya enseñanzas que puedan requerir tres años y otras cuatro pero, plantea que se valore la exigencia de que un mismo título tenga la misma duración en todas las universidades. De hecho, en el dictamen se dice que parece difícil alcanzar la homogeneización con los países del entorno, perseguida con la modificación, cuando dentro de nuestro propio país podría darse el caso de que, un mismo título tuviera una duración diferente en una universidad y otra. El dictamen del Consejo de Estado también recomendaba un plazo más largo para que la adaptación al nuevo sistema fuese más coordinada por las universidades. Finalmente, se hacía referencia a la ambigüedad en lo relativo a los títulos que habilitan para el acceso a profesiones reguladas o, incluso grados no habilitantes pero que son condiciones de acceso a másteres habilitantes. El Consejo de Estado advierte que no en todas las normas sectoriales actuales se establece expresamente la duración de los estudios, e incluso hay profesiones pendientes de ser reguladas.

El PSOE se basó en este dictamen del Consejo de Estado para presentar en el Congreso de los Diputados una proposición no de ley en la que solicitaban que el Decreto no se

\footnotetext{
${ }^{6}$ http://www.boe.es/buscar/doc.php?id=CE-D-2014-1146
} 
aprobara sin un informe previo sobre los resultados del sistema actual y una evaluación de una comisión mixta con presencia de comunidades autónomas, rectores y estudiantes.

En general, se podría decir que las universidades no se oponen a un cambio en la estructuración de las titulaciones pero consideran que, no se dan las condiciones necesarias ni la oportunidad para implantar ahora un modelo distinto, aunque creen conveniente y de interés, el estudio de forma amplia, serena y rigurosa sobre cuál puede ser la mejor estructura de las titulaciones universitarias. Consideran que, se cambió a un nuevo modelo, de forma generalizada, en 2010 para adaptarse al EEES y dicho cambio aún no ha sido convenientemente valorado y analizado. Así se desprendía ya en un informe de la Conferencia de Rectores de Universidades Españolas (CRUE) de julio de 2014. También lamentaban la poca receptividad del Ministerio a las sugerencias planteadas por las Universidades y recogían una serie de aspectos que preocupaban de la reforma, entre ellos la coexistencia de grados de 180 y 240 créditos con la misma denominación y no se sabe con qué competencias, dudas sobre quién decidirá la duración y competencias profesionales de los grados, e incluso dudas sobre el reconocimiento por parte de empleadores y colegios profesionales de títulos con diferentes duraciones y el acceso al mercado laboral por parte de los nuevos graduados con un menor tiempo de formación. Otros temas que preocupan a las universidades son los accesos a másteres con competencias profesionales con grados de 3 años. Las universidades consideran que en la práctica los estudiantes se verán obligados a cursar Grado+Máster, con el correspondiente coste social. Otro aspecto también advertido por las universidades es la saturación de las Agencias de Calidad, con acreditaciones de títulos actuales y verificaciones de los nuevos.

La CASUE (Comisión Académica Sectorial de Universidades Españolas) también alerta sobre "la sensación de confusión que puede producir en los empleadores y sociedad en general: actualmente conviven el sistema pre-Bolonia, con titulaciones de cinco años, con el nuevo sistema de cuatro años. Introducir un tercer sistema, cuando no está asentado el actual puede resultar muy confuso".

Finalmente la CRUE, ante la inminente aprobación del Decreto, reunida en Asamblea General el 2 de febrero de 2015, acordó (con 57 votos a favor, cinco abstenciones y un voto en contra) reafirmarse en la necesidad de una moratoria para la aplicación de la modificación y solicitar a todas las universidades y comunidades autónomas que no inicien la tramitación de nuevas titulaciones hasta septiembre de 2016 (oferta de nuevos Grados a partir del curso 2017/2018). La CRUE vuelve a insistir en priorizar la evaluación de las titulaciones vigentes 
y no realizar cambios de manera precipitada; destaca la necesidad de una profunda reflexión sobre el futuro de la universidad española y su modelo de financiación. La CRUE declara, como su principal preocupación la defensa de la calidad de la oferta académica, la igualdad de oportunidades para los estudiantes y asegurar la mejor formación y empleabilidad de los egresados.

Además de los pronunciamientos de la CRUE, son también muchos los rectores que han calificado la reforma de inoportuna, prematura o precipitada, incluso rectores de universidades que en su momento se mostraban abiertamente partidarias de un modelo $3+2$. La mayoría de los rectores piden un debate sosegado y amplio, con la necesidad de evaluar determinados aspectos del modelo actual: nuevas competencias que están adquiriendo los graduados, su empleabilidad, el rendimiento académico, diversidad de la oferta académica, capacidad de atracción de estudiantes por las universidades españolas,... Por parte de las universidades públicas se recuerda también los fuertes recortes en los presupuestos que se han producido los últimos años.

Por parte de los representantes y asociaciones estudiantiles se comparten muchas de las preocupaciones de los Rectores. Analizando las declaraciones e informes emitidos por la Conferencia de Representantes de Estudiantes de Universidades Públicas (CREUP) ${ }^{7}$, por la organización Estudiantes en Movimiento ${ }^{8}$ o por el Sindicato de Estudiantes, se podría hacer un listado de los argumentos esgrimidos por los representantes estudiantiles para rechazar la reforma en este momento o plantear objeciones sobre su oportunidad. Algunos de los cuales han sido también utilizados por los Rectores. Destacan que los objetivos de empleabilidad, movilidad y especialización que prometía el proceso de Bolonia están lejos de cumplirse. Critican la actitud impositiva del Gobierno y la falta de voluntad para negociar con el colectivo estudiantil en el diseño de Políticas Universitarias, y valoran positivamente cualquier debate sobre la oferta académica y una posible reestructuración de los títulos universitarios pero, con tiempo, argumentos y diálogo suficiente. Los estudiantes muestran su preocupación por la coexistencia de 3 sistemas distintos, la devaluación de los grados de 180 créditos ECTS y mayores dificultades para el acceso al mercado laboral, y el encarecimiento de los estudios y, preocupación por la igualdad de oportunidades ante los costes de los Másteres. Para obtener una formación adecuada para el mercado laboral consideran que habrá que pagar dos años

\footnotetext{
${ }^{7}$ http://www.creup.es/wp-content/uploads/2014/09/INFORME-DE-CREUP-SOBRE-LA-PROPUESTADE-REESTRUCTURACI\%C3\%93N-DE-LOS-T\%C3\%8DTULOS-UNIVERSITARIOS-Y-LA-INCLUSI \%C3\%93N-DE-GRADOS-DE-180-ECTS_p.pdf

${ }^{8} \mathrm{http://estudiantesenmovimiento.org/Pp}=532$
} 
de máster a precios inasumibles para la mayoría. Finalmente, los estudiantes manifiestan su temor a la supresión de Grados y el despido de profesorado.

Esta preocupación por el posible efecto en forma de recortes de profesorado en las universidades públicas también ha sido advertida por parte de las organizaciones sindicales. Las organizaciones sindicales también han querido poner de manifiesto su preocupación por diferentes aspectos: la realización de una reforma sin el consenso en la comunidad universitaria, la preocupación por lo que a su juicio es un objetivo de convertir las universidades en empresas de servicios educativos; el encarecimiento de los estudios universitarios, expulsando del sistema al alumnado con menos poder adquisitivo; los elevados precios de los estudios de postgrado y las mayores dificultades para el acceso a becas; el impacto negativo en la financiación de las universidades. Los sindicatos comparten también con los Rectores la necesidad de evaluar antes los Grados que comenzaron a impartirse a raíz del Decreto de 2007.

Diversas voces han planteado su preferencia por el modelo " $3+2$ " frente al " $4+1$ ". Las universidades catalanas lo defendieron antes incluso del aprobar el Real Decreto 1393/2007 y, por tanto, no están en contra de algo que en su día apoyaron, pero reconocen la necesidad de más debate y análisis del sistema actual. También, exigen no aumentar los precios de las matrículas. La Secretaría Catalana de Universidades defiende el cambio como algo necesario si queremos explorar el mercado internacional. La secretaría también pide más debate, pero recuerda que en Europa existen países donde cohabitan ambos sistemas.

La Conferencia de Consejos Sociales de las Universidades cree que un sistema dual "no solamente es viable, simo bueno", y lo considera una "pequeña modificación" que ya recogía el informe de expertos de Wert. Señala que hay que ir más allá y pide una reforma estructural profunda del mapa universitario.

Responsables del U-Ranking ${ }^{9}$ elaborado por la Fundación BBVA y el Instituto Valenciano de Investigaciones Económicas (Ivie) también han manifestado recientemente su apoyo a la reforma que permite la reducción de los Grados, pero alertan de que tendría efectos negativos si no se acompaña con becas.

También, entre la comunidad universitaria, pueden encontrarse opiniones que, reconocen la infravaloración en algunos casos de los másteres de 60 créditos, y la dificultad en algunas áreas para lograr verdaderas especializaciones con programas formativos en los que, descontando el trabajo fin de Máster y las prácticas externas, el número de créditos

\footnotetext{
${ }^{9}$ http://www.u-ranking.es/
} 
formativos es muy reducido. Asimismo, se reconoce la sobredimensionada oferta actual de titulaciones y la necesidad de racionalizarla. En el caso de los Grados hay voces que, consideran que su conversión en titulaciones de 4 años hizo en su momento que, en algunas disciplinas se añadieran créditos no estrictamente necesarios para lograr las competencias exigidas o, se aumentaran de manera excesiva los créditos de prácticas externas.

\section{Implicaciones económicas de la modificación de la estructura de las titulaciones}

En primer lugar, debe recordarse que el Real Decreto 43/2015 incorpora una disposición adicional que establece que las medidas incluidas en el mismo no podrán suponer incremento de dotaciones ni de retribuciones ni de otros gastos de personal. Sin embargo, en los argumentos utilizados por la comunidad universitaria aparecen algunos aspectos en los que hay discrepancias con el Ministerio sobre las implicaciones económicas de la reforma: En concreto el posible impacto negativo en la financiación de las universidades públicas y la posibilidad de que se planteen recortes en profesorado; y las consecuencias en las economías familiares de la reducción de un año en los Grados.

Respecto al primer punto, los sindicatos y los rectores ven que este decreto puede suponer una reducción de personal porque mientras los Grados son más "generalistas" y permiten una asignación más flexible de docentes, el Máster requiere más especialización. En cualquier caso, el Ministerio promete que la reforma "no supondrá despido de profesorado universitario" porque "la práctica totalidad del profesorado que en estos momentos está impartiendo grados está capacitado para impartir también másteres".

Por otro lado, el propio Ministerio asegura que en "el 90\% de las carreras no hay que hacer un máster de manera obligatoria para poder trabajar" y estima que sólo el $20 \%$ de los estudiantes pasa del Grado al Máster y que lo hace generalmente tras haberse incorporado al mercado laboral. De ser ciertas las estimaciones del Ministerio la reforma ocasionaría un descenso de los estudiantes en las universidades (estarían un año menos) y se hace comprensible la preocupación de las organizaciones sindicales y los rectores por el posible efecto en las plantillas de profesorado.

Respecto a la estimación que hace el Ministerio de un ahorro de 150 millones de euros se basa en que se cursa un año menos de Grado y la no necesidad de cursar un Máster, en la mayoría de los casos. Obviamente, los costes de los Grados se reducirían en cuantías equivalentes al coste de un curso, pero esa cantidad, en el caso de las universidades públicas, sería diferente en función de la Comunidad Autónoma. Tomando como referencia los precios 
del curso académico 2013-14, los ahorros irían desde los 713,40 € de Galicia a los 2.011,20€ de Cataluña. Pero también parece lógico realizar un análisis global de los costes de formación, incluyendo el Máster. Generalizar el modelo de Máster de 2 años supondría también un coste extra para los estudiantes. De manera que, si globalmente se comparan los modelos $4+1$ y $3+2$ el coste total se incrementaría, tal como puede verse en la Tabla 6 .

\begin{tabular}{lrrrrrr}
\hline & \multicolumn{3}{c}{ Con Máster habilitante } & \multicolumn{2}{c}{ Con Máster no habilitante } \\
\hline & \multicolumn{1}{c}{$4+1$} & \multicolumn{1}{c}{$3+2$} & Diferencia & \multicolumn{1}{c}{$4+1$} & \multicolumn{1}{c}{$3+2$} & Diferencia \\
\hline Precio medio & $6.034,20$ & $6.542,40$ & 508,20 & $6.827,40$ & $8.128,80$ & $1.301,40$ \\
\hline Andalucía & $4.198,80$ & $4.611,60$ & 412,80 & $5.518,80$ & $7.251,60$ & $1.732,80$ \\
\hline Aragón & $5.928,00$ & $5.931,00$ & 3,00 & $7.492,80$ & $9.060,60$ & $1.567,80$ \\
\hline Asturias (Ppdo. de) & $5.703,60$ & $6.268,20$ & 564,60 & $6.118,20$ & $7.097,40$ & 979,20 \\
\hline Balears (Illes) & $5.997,60$ & $6.619,20$ & 621,60 & $6.137,40$ & $6.898,80$ & 761,40 \\
\hline Canarias & $5.265,60$ & $5.968,20$ & 702,60 & $5.759,40$ & $6.955,80$ & $1.196,40$ \\
\hline Cantabria & $4.611,00$ & $5.172,00$ & 561,00 & $5.433,60$ & $6.817,20$ & $1.383,60$ \\
\hline Castilla y León & $7.493,40$ & $8.053,80$ & 560,40 & $8.016,60$ & $9.100,20$ & $1.083,60$ \\
\hline Castilla-La Mancha & $5.316,00$ & $6.060,00$ & 744,00 & $5.412,60$ & $6.253,20$ & 840,60 \\
\hline Cataluña & $10.497,60$ & $10.939,20$ & 441,60 & $11.969,40$ & $13.882,80$ & $1.913,40$ \\
\hline Com. Valenciana & $6.117,00$ & $6.117,00$ & 0,00 & $7.665,60$ & $9.214,20$ & $1.548,60$ \\
\hline Extremadura & $4.837,80$ & $5.253,60$ & 415,80 & $5.502,60$ & $6.583,20$ & $1.080,60$ \\
\hline Galicia & $4.355,40$ & $5.143,80$ & 788,40 & $4.443,00$ & $5.319,00$ & 876,00 \\
\hline Madrid (Com. de) & $9.844,20$ & $10.589,40$ & 745,20 & $11.179,20$ & $13.259,40$ & $2.080,20$ \\
\hline Murcia (Región de) & $5.271,00$ & $5.868,00$ & 597,00 & $6.392,40$ & $8.110,80$ & $1.718,40$ \\
\hline Navarra (C. Foral) & $6.274,80$ & $6.783,60$ & 508,80 & $7.272,00$ & $8.778,00$ & $1.506,00$ \\
\hline País Vasco & $5.094,00$ & $5.145,00$ & 51,00 & $5.931,00$ & $6.819,00$ & 888,00 \\
\hline Rioja (La) & $5.586,00$ & $5.661,00$ & 75,00 & $6.780,00$ & $8.049,00$ & $1.269,00$ \\
\hline U.N.E.D. & $6.216,60$ & $7.579,20$ & $1.362,60$ & $5.859,00$ & $6.864,00$ & $1.005,00$ \\
\hline
\end{tabular}

Tabla 6: Diferencias en los precios públicos en Universidades Públicas comparando los modelos " $4+1$ ” y " $3+2$ ".

Tomando como referencia el número actual de estudiantes de las universidades públicas españolas y, de cumplirse las previsiones del Ministerio de que únicamente un 20\% de los graduados optarían por cursar un Máster, considerando además como precio del Máster el promedio entre los habilitantes y no habilitantes, el ahorro global en tasas de matrícula se podría acercar a los 850 millones de euros al pasar al modelo “3+2". Ese cálculo se muestra en la Tabla 7.

\begin{tabular}{lrrr}
\hline & $\begin{array}{r}\text { Tasa de continuación } \\
\text { del } 20 \%\end{array}$ & $\begin{array}{r}\text { Tasa de continuación } \\
\text { del } 50 \%\end{array}$ & $\begin{array}{r}\text { Tasa de continuación } \\
\text { del } 75 \%\end{array}$ \\
\hline Número de estudiantes de Grado & 1.200 .000 & 1.200 .000 & 1.200 .000 \\
\hline Número de estudiantes de Máster & 240.000 & 600.000 & 900.000 \\
\hline Coste global del modelo 4+1 & $5.787,36$ millones $€$ & $6.510,96$ millones $€$ & $7.113,96$ millones $€$ \\
\hline Coste global del modelo 3+2 & $4.943,52$ millones $€$ & $6.390,72$ millones $€$ & $7.596,72$ millones $€$ \\
\hline Diferencia & 843,84 millones $€$ & 120,24 millones $€$ & $-482,76$ millones $€$ \\
\hline
\end{tabular}

Tabla 7: Estimación de los importes totales de matrícula en las universidades públicas con diferentes porcentajes estimados de estudiantes de Grado que continúan posteriormente el Máster. 
Realmente el escenario planteado por el Ministerio generaría incluso más ahorros, ya que considera el 20\% sobre los graduados y no sobre el total de estudiantes de Grado, por lo que en el cálculo anterior se estaría asumiendo una tasa de graduación del 100\%. En ese escenario puede tener sentido la afirmación del Ministerio de generar un ahorro para las familias de 150 millones, pero lo cierto es que esos valores de tasas de continuación de estudios en un nivel superior son sensiblemente menores a los de países de nuestro entorno. Además, muchas voces plantean que grados más cortos tendrían una inserción laboral menor y que aumentaría el número de graduados que se verían obligados a cursar un Máster. Si se considera tasas de continuación del 50\%, como el Informe de la EACEA estima para países como Alemania, el ahorro sería de apenas 120 millones de euros. Y, si se toman tasas de continuación del 75\%, de países como Francia, la diferencia sería negativa, con un incremento en los costes totales de matrícula de casi 500 millones de euros. En realidad, basta con que un 55\% de los estudiantes de Grado opte para continuar estudiando un Máster para que el nuevo modelo tenga un coste mayor en precios públicos de matrícula

\section{Conclusiones}

En este trabajo se ha tratado de reflejar datos y recoger las diferentes opiniones que la reciente modificación de la estructura de las titulaciones oficiales ha generado en la comunidad universitaria, con vistas a servir de punto de partida para un debate sosegado sobre la oportunidad de la reforma y el análisis de posibles medidas que deberían acompañar a esta propuesta.

Estudios previos a la puesta en marcha del Plan Bolonia parece que venían a incidir que ya existían diferencias de clase a la hora de elegir carreras universitarias; los estudiantes provenientes de clases trabajadoras apostaban mucho más a menudo por carreras de tres años, lo que entonces eran diplomaturas, títulos de maestros o ingenierías y arquitectura técnicas. Incluso estudios de algunas universidades constataron una reducción de nuevos estudiantes de clase trabajadora con los nuevos planes de estudio de cuatro años ${ }^{10}$. Desde ese punto de vista los grados de tres años pueden beneficiar a estudiantes de clase trabajadora, pero también es cierto que voces críticas advierten del riesgo de que los grados se conviertan en una prolongación del bachillerato y que la verdadera formación para el empleo se vaya trasladando a los postgrados. En ese sentido parece que, hay unanimidad en la comunidad

\footnotetext{
${ }^{10} \mathrm{http}: / /$ grupsderecerca.uab.cat/gret/content/el-impacto-de-la-desaparici\%C3\%B3n-de-las-titulaciones-deciclo-corto-tras-la-reforma-de-bolonia
} 
universitaria en exigir que la reforma vaya acompañada de una política de becas que favorezca la igualdad de oportunidades y que no limite la posibilidad de acceso a los estudios de Máster a estudiantes con condiciones socioeconómicas peores.

También parece que hay bastante consenso en que la forma de aprobar esta modificación no ha sido la adecuada, que cambios tan profundos y de tanto calado deben venir acompañados de un mayor acuerdo, tal como reclamaba el Consejo de Estado. Otro punto de coincidencia es la necesidad de hacer una valoración seria y sosegada de los resultados que se están consiguiendo con la reforma iniciada de la puesta en marcha de los primeros Grados adaptados al EEES.

La preocupación entre la comunidad universitaria por las implicaciones económicas de la reforma también es comprensible. Por un lado, en la actual situación de los másteres, con tasas académicas sensiblemente más altas y requisitos académicos para el acceso a becas también más altos, se justifica la preocupación por llegar a una situación de estudiantes de "primera" y estudiantes de "segunda" en función de las posibilidades de las familias para acceder a la formación de nivel de Máster. También es entendible la preocupación de las universidades públicas por los efectos en su financiación; aunque tendrán autonomía para configurar grados de 3 o 4 años, la duda surge al plantearse cómo las Comunidades Autónomas financiarán a sus universidades.

Finalmente sería deseable alcanzar un consenso y dotar a nuestro sistema universitario de una estabilidad que le permita centrar sus esfuerzos en la búsqueda de la excelencia en la formación de los futuros profesionales. Los constantes cambios que se vienen produciendo en los últimos años en el sistema universitario español están generando una situación de estrés y un cierto desánimo entre la comunidad universitaria. Además parece obvio que cualquier modificación en una de las etapas del sistema educativo debería ser fruto de un análisis global del sistema y no acometer reformas independientes en cada uno de los niveles sin valorar las implicaciones que tienen para la formación global de los estudiantes. 